\title{
Detection and distribution of aluminium in bone
}

\author{
JOHN DENTON, ANTHONY J FREEMONT, JOHN BALL \\ From the Department of Rheumatology, University of Manchester Medical School, Stopford Building, \\ Oxford Road, Manchester M13 9PT.
}

SUMMARY The distribution of aluminium has been investigated using a new histochemical technique in transiliac bone biopsy specimens from 20 patients with aluminium related bone disease and 17 with other metabolic bone diseases. The method, which employs solochrome azurine at acid $\mathrm{pH}$ (acid solochrome azurine), showed a wider distribution of aluminium within cortical and trabecular bone than other histochemical techniques. The results compared well with atomic absorption spectrophotometric analysis of these biopsy specimens. Wavelength dispersive electron probe analysis of serial sections validated the procedure. The method has certain advantages over both these analytical techniques.

The presence of aluminium in the tissues of patients undergoing chronic haemodialysis is an important cause of morbidity and mortality,' especially in areas where water authorities use Alum to clear particulate matter from domestic supplies. The deposition of aluminium in bone is associated with a depression of osteoblastic and osteoclastic activity, ${ }^{2}$ a condition known as dialytic bone disease. Two histochemical methods, employing aluminon (ammonium aurine tricarboxylic acid) or solochrome azurine, have been used to detect this metal in undecalcified sections of bone biopsy specimens from patients with dialytic bone disease and both have shown aluminium at the calcification fronts between osteoid and bone. ${ }^{34}$ This would indicate that aluminium is being incorporated into the crystal lattice, but neither method reliably detects aluminium deeper within bone. One explanation for this apparent anomaly is that the methods referred to detect only ions which are immediately available for exchange, such as those at the fronts where mineralisation is incomplete. With the achievement of full mineralisation ionic exchange reactions become progressively less likely ${ }^{5}$ and any aluminium present may presumably be less available for reaction with the dye. We have therefore developed an alternative histochemical method for demonstrating aluminium which takes this into account and shows aluminium at the fronts and elsewhere in the bone.

A problem with histochemical techniques for detecting aluminium is that they are not completely specific. ${ }^{6}$ We have examined the specificity of our method and others using a wide range of known Accepted for publication 7 November 1983 metals in a paper spot test procedure. We have also compared the results for aluminium content and distribution obtained using our technique with those obtained by wavelength dispersive electron probe analytical microscopy and atomic absorption spectrophotometry.

\section{Material and methods}

TISSUE SPECIMENS AND HISTOCHEMICAL

TECHNIQUES

Transiliac core biopsy specimens, $4 \mathrm{~mm}$ in diameter, from 37 patients were studied. Twenty patients had been receiving chronic haemodialysis for between one and 10 years, and of the remaining 17 , who had no clinical or biochemical evidence of renal failure, six had osteoporosis, six vitamin D deficiency osteomalacia, and five primary hyperparathyroidism as judged by clinical, radiological, and biochemical criteria.

Biopsy specimens were fixed in AR ethanol, double embedded in low viscosity nitrocellulose and wax, ${ }^{7}$ and $10 \mu \mathrm{m}$ undecalcified tissue sections prepared. (All methods are negative in decalcified sections.) Six of the blocks from patients receiving haemodialysis were dewaxed and re-embedded in various methacrylate based resins: two were embedded in a mixture of $20 \% \mathrm{wt} / \mathrm{vol}$ polyethylene glycol distearate and $80 \% \mathrm{wt} / \mathrm{vol}$ methylmethacrylate (PEGM), two methylmethacrylate alone, and two in a mixture of $30 \%$ methymethacrylate and $70 \%$ butylmethacrylate. Further $10 \mu \mathrm{m}$ sections were prepared from these blocks.

A sample of the cancellous bone was removed 
from all the blocks and sent to the department of chemistry, where it was analysed for aluminium using atomic absorption spectrophotometry.

Sections from all blocks were stained using our method (method 1). In 10 unselected haemodialysis cases the aluminon (method 2) and alkaline (pH8) solochrome azurine (method 3) techniques were used to stain serial sections. The aluminon and alkaline solochrome azurine solutions were prepared and used as described by Pearce. ${ }^{6}$

In method 1 a $1 \%$ aqueous solochrome azurine (RA Lamb CI43830) stock solution was adjusted to pH5 with $25 \% \mathrm{vol} / \mathrm{vol}$ glacial acetic acid in water. The fine flocculent precipitate formed was not filtered, and the stain was used immediately. The $10 \mu \mathrm{m}$ undecalcified sections were taken to water and stained for $18 \mathrm{~h}$, differentiated in $95 \%$ alcohol until the section was a pale pink colour (5-10 min), counterstained with a $1 \%$ solution of safranin, dehydrated, and mounted in XAM (Gurr). Sections prepared from methacrylate embedded tissue were stained in exactly the same way either still embedded in the resin or, in the case of PEGM embedded sections, after the resin had been removed by immersion in chloroform for $10 \mathrm{~min}$.

The working limits of the technique have been established on double embedded blocks as follows:

(i) Precipitate. The effects on staining of filtering the precipitate, and its behaviour if left undisturbed for many hours, were observed.

(ii) Staining time. Fifteen sections from the same case were removed from the staining solution at two hourly intervals from two to 30 hours and were processed and compared.

(iii) Differentiation time. Seven sections were each stained for $18 \mathrm{~h}$ and then placed in $95 \%$ alcohol. Sections were removed, counterstained, and mounted at $1,5,10,15,20,30$, and $60 \mathrm{~min}$.

(iv) Reproducibility. Four serial sections from biopsy specimens from each of two patients receiving chronic haemodialysis were processed on four consecutive days, on each occasion using a freshly prepared dye solution.

The results of these studies are given below, but the optimal staining practices are those specified in the above protocol.

\section{PAPER SPOT TEST}

Aqueous solutions (usually of chloride salts) of each of 25 different metals (lithium, beryllium, boron, magnesium, aluminium, calcium, titanium, vanadium, chromium, manganese, iron, cobalt, nickel, copper, zinc, arsenic, selenium, tin, molybdenum, cadmium, strontium, barium, mercury, lead, and bismuth) were applied to filter paper to form spots of similar diameter each containing $0.1 \mathrm{mg}$ of the salt. The filter papers were then stained for $5 \mathrm{~min}$ by each of the three methods, differentiated as if they were tissue sections, and air dried.

\section{ATOMIC ABSORPTION SPECTROPHOTOMETRY}

All the specimens were analysed for the presence of aluminium using a previously described atomic absorption spectrophotometric technique. ${ }^{8}$ Biopsy specimens from five of the haemodialysis patients were also examined for beryllium, titanium, chromium, and copper.

\section{ELECTRON PROBE ANALYSIS}

Wavelength dispersive electron probe point analytical microscopy (which is more sensitive than its energy dispersive equivalent) is capable of detecting the presence of elements of atomic number greater than five in tissue sections providing they are present in high enough concentrations. The microscope (CAMECA Camabax) operates by scanning a narrow track across a tissue section, semiquantitatively analysing $1 \mu \mathrm{m}$ square areas for the presence of a predetermined element. The track leaves a trace on the section which can be recorded photographically, and the results are compared with histochemical stains performed on serial sections.

Undecalcified unstained sections $(15 \mu \mathrm{m})$ from nine haemodialysis patients were fixed to quartz slabs with Araldite, vacuum sputter-coated with carbon, and photographed after suitable areas had been chosen for study. After analysis the sections were again photographed. All nine sections were analysed for aluminium, calcium, and phosphorus, and two were also analysed for iron, titanium, and copper. The results from this analysis were also compared with serial sections stained by method 1 .

Three $15 \mu \mathrm{m}$ undecalcified sections were stained and differentiated by method 1 before examination in the probe for the presence of calcium, aluminium, and phosphorus.

\section{OTHER HISTOCHEMICAL TECHNIQUES}

As the acid solochrome azurine method (method 1) also detects iron (see results section) sections from each of the 37 biopsy specimens were stained with Perl's reagent and compared with adjacent sections stained by method 1 .

Sections from each biopsy specimen were also stained by von Kossa's method to distinguish osteoid from bone.

\section{Results}

With method 2 staining indicative of aluminium was 
restricted to a linear zone at the bone surfaces mainly between osteoid and bone. With method 3 a similar pattern was seen, but occasional cement lines method 1 often showed positive blue staining deep in trabecular and cortical bone (Fig. 1) as well as the fronts and cement lines, but not in uncalcified osteoid. The density and position of this deep blue reaction product varied considerably from patient to patient and between cortical and trabecular bone, but as a general rule it was distributed linearly within bone in a pattern which corresponded to cement lines in trabeculae and concentrically about Haversian canals in the cortex. Elsewhere in the bone the stain was much less prominent, but, particularly when the linear stain was dense, more diffuse staining could be detected between cement lines. This did not appear to be an overstaining artefact as it was often absent from the centre of trabeculae and areas of cortex furthest from Haversian systems (Fig. 1).

Staining was graded as follows: $(+)=$ stain restricted to the fronts, $(++)=$ stain at both the fronts and cement lines with little or no diffuse staining, and $(+++)$ and $(++++)$ as for $(++)$ but with an increasing amount of diffuse staining (Fig. 2). Staining in osteocytes and cannaliculi was sometimes seen in grade $(++++)$ reactions. As a rule osteoblasts and osteoclasts showed little or no staining, but stain was sometimes seen within bone marrow cells but not in the connective tissue in marrow or attached to bone. When the staining reaction was compared in sections from blocks embedded in various media the results were essentially the same.

The results obtained by applying this scoring sysalso stained. By contrast, in the same specimens

tem to each of the 37 biopsy specimens are shown in Table 1, where they are also compared with the quantity of aluminium found by atomic absorption spectrophotometry. All 20 biopsy specimens from dialysis patients contained aluminium in a range of $16-406 \mu \mathrm{g} / \mathrm{g}$ (mean $141 \mu \mathrm{g} / \mathrm{g}$ ) of dried anorganic trabecular bone devoid of marrow, compared with $0-22 \mu \mathrm{g} / \mathrm{g}$ (mean $8 \mu \mathrm{g} / \mathrm{g}$ ) for the other metabolic bone disorders. Table 1 shows that within the limits of the technique there were no false positive or negative results and there was some correlation between the subjective assessment of aluminium and that derived by spectrophotometry, although there was overlap between groups.

When wavelength dispersive electron probe analysis was applied to unstained sections from patients receiving haemodialysis, calcium and phosphorus, as expected, were seen throughout the bone. Peaks of high concentration were sometimes seen, some of which were related to cement lines, which are known to have a higher mineral concentration than surrounding bone. ${ }^{5}$ The distribution of aluminium tended to follow that of calcium, with prominent peaks at the osteoid bone junctions and to a lesser extent in cement lines. Elsewhere within the bone much less or no aluminium was detected (Fig. 3). The distribution of aluminium shown by this technique was similar to the patterns of the reaction product in serial sections stained with acid solochrome azurine. Sections stained by method 1 before examination with the electron probe contained no calcium but peaks of aluminium could be seen and these corresponded to the areas of greatest stain density (Fig. 4).

When the specificity of the three methods was

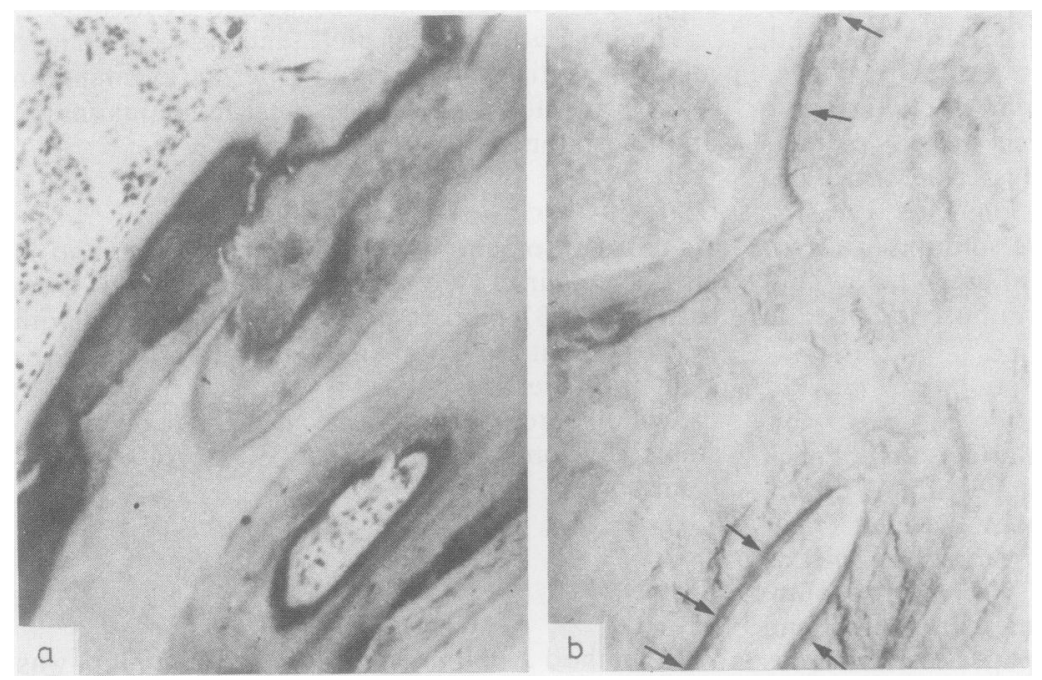

Fig. 1 Serial sections of the same area of cortical bone. With method 1 (a) degrees of staining are seen except in the central part and the osteoid. With method 2 (b) staining is restricted to a thin linear zone between osteoid and bone (arrowed). Original magnification (a) $\times 100$, (b) $\times 200$. 


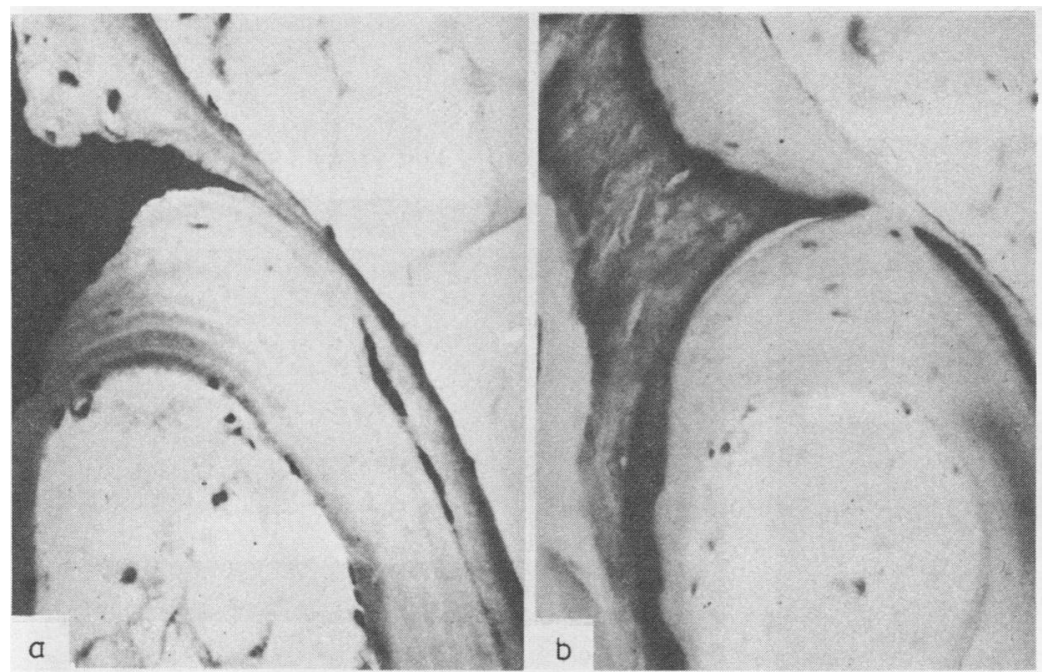

Fig. 2 Serial sections of a trabeculum stained by von Kossa method (a) and method 1 (b). The osteoid does not stain with method 1 and, although staining is present throughout the calcified bone, it is most dense at the fronts. Original magnification $\times 150$.

compared in the spot test considerable variation was found (Table 2). None of the dyes reacted with the salts of boron, magnesium, vanadium, arsenic, selenium, tin, molybdenum, strontium, barium, mercury, or bismuth. Aluminon (method 2) was the most specific, reacting with only beryllium, aluminium, and iron; and the reaction with iron gave a different colour from the others. The alkaline solochrome azurine (method 3) was the least specific,

Table 1 Relation between the scoring system based on method 1 and the quantities of aluminium (per gram of anorganic trabecular bone) found by atomic absorption spectrophotometry

\begin{tabular}{|c|c|c|c|}
\hline \multicolumn{2}{|c|}{ Haemodialysis patients } & \multicolumn{2}{|c|}{ Non-haemodialysis patients } \\
\hline $\begin{array}{l}\text { Stain } \\
\text { score }\end{array}$ & $\begin{array}{l}\text { Bone aluminium } \\
(\mu \mathrm{g} / \mathrm{g})\end{array}$ & $\begin{array}{l}\text { Stain } \\
\text { score }\end{array}$ & $\begin{array}{l}\text { Bone aluminium } \\
(\mu \mathrm{g} / \mathrm{g})\end{array}$ \\
\hline $\begin{array}{r}+ \\
+ \\
+ \\
+ \\
+ \\
+ \\
+ \\
+ \\
++ \\
++ \\
++ \\
++ \\
++ \\
++ \\
++ \\
++ \\
+++ \\
+++ \\
+++ \\
+++ \\
++++\end{array}$ & $\begin{array}{r}16 \\
22 \\
22 \\
30 \\
44 \\
72 \\
29 \\
55 \\
58 \\
62 \\
80 \\
87 \\
133 \\
141 \\
254 \\
166 \\
173 \\
215 \\
245 \\
406\end{array}$ & $\begin{array}{l}- \\
- \\
- \\
- \\
- \\
- \\
- \\
- \\
- \\
- \\
- \\
- \\
- \\
- \\
- \\
- \\
-\end{array}$ & $\begin{array}{r}0 \\
1 \\
1 \\
2 \\
2 \\
3 \\
3 \\
4 \\
4 \\
4 \\
6 \\
7 \\
15 \\
18 \\
21 \\
22 \\
22\end{array}$ \\
\hline
\end{tabular}

$+=$ stain restricted to the fronts; $++=$ stain at both the fronts and the cement lines with little or no diffuse staining; +++ and ++++ $=$ as for ++ but with an increasing amount of diffuse staining; $-=$ no stain. but the range of colours of the products made it relatively easy to distinguish the metals concerned. After differentiation, acid solochrome azurine reacted with six metals-beryllium, aluminium, titanium, copper, chromium, and iron-and in every case the product was a variant of blue.

With the atomic absorption spectrophotometric technique it was impossible to show beryllium, titanium, copper, or chromium in trabecular bone; nor were iron, titanium, or copper found in the specimens examined using the electron probe, and in none of the 37 biopsies was a positive result obtained with the Perl's method in bone trabeculae even in the presence of heavy staining in the marrow (Fig. 5).

The practical limits of the technique presented no problems. The flocculent material remained in suspension for $18 \mathrm{~h}$ but after this time started to precipitate out, a process completed by $24 \mathrm{~h}$. Neither the supernatant from the spontaneously precipitated solution or that from filtered, newly prepared dye produced a staining reaction.

The electron probe showed that during the staining period method 1 decalcifies the section, and the end point of this process is the lower limit of staining time. Until full decalcification has occurred, which for a $10 \mu \mathrm{m}$ thick section takes between 14 and $16 \mathrm{~h}$ at room temperature, the remaining calcium stains an orange red colour which largely masks the blue reaction product. Once decalcification is complete the blue staining does not increase in density.

Providing decalcification was complete differentiation to give a pale pink background took less than $10 \mathrm{~min}$. If necessary the section could be left for as long as $60 \mathrm{~min}$ in the alcohol without a noticeable change in the blue staining. 

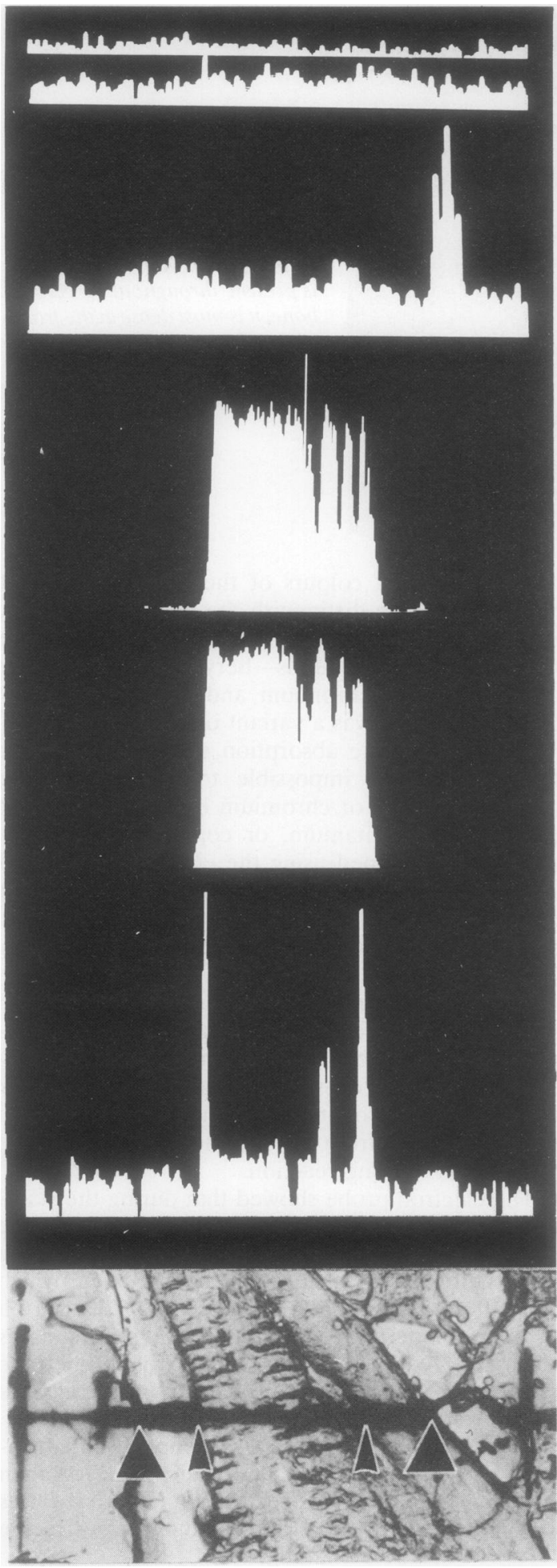

Denton, Freemont, Ball

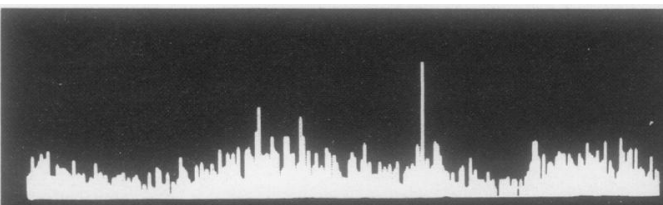

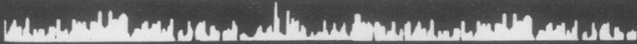
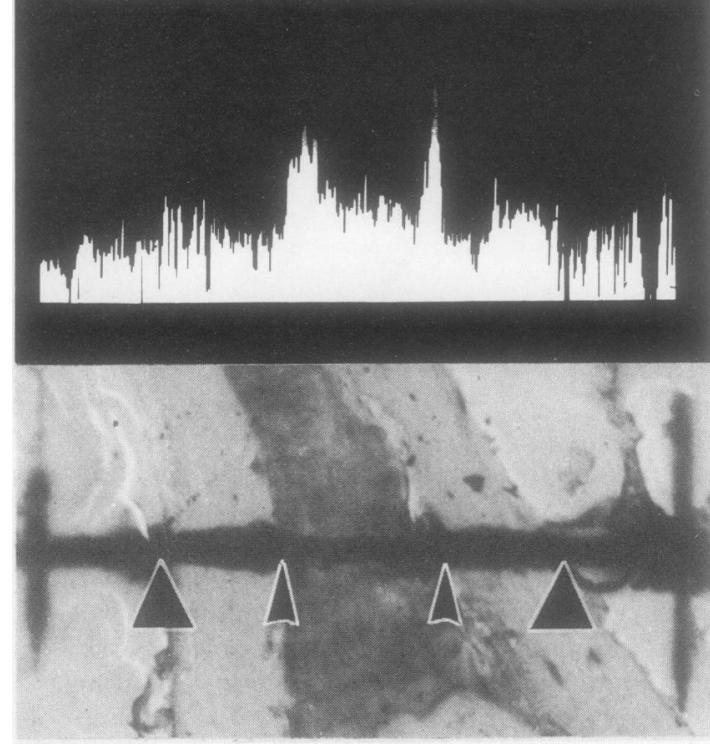

Fig. 4 Serial section to that in fig. 3, stained by method 1 before electron probe analysis. All traces are to the same scale. From above downwards the traces are for phosphorous, calcium, and aluminium. Peaks of aluminium correspond with the fronts. The method also stains bone in the centre of the trabeculum, but the plot barely distinguishes this area from background, indicating the relative sensitivity of method 1 . As the method decalcifies during processing the amounts of calcium and phosphorous detected by the probe over bone are no higher than background. Original magnification $\times 150$.

Fig. 3 Iliac crest biopsy specimen from a patient receiving haemodialysis. The track made by the probe is seen across the unstained trabeculum, delineated by large arrows; the small arrows indicate junction of osteoid and bone. From above downwards the plots represent analyses along that track for titanium, copper, iron, calcium, phosphorous, and aluminium. The vertical scale varies such that $1 \mathrm{~cm}$ represents 1 unit for titanium, copper, and iron, 100 units for calcium, 20 units for phosphorous, and 3 units for aluminium. Aluminium is confined to the mineralised bone in which copper, iron, and titanium are not demonstrable. $A$ peak for iron is seen over a cellular area of marrow. 
Table 2 Results of the filter paper spot test for the various positive metal ions stained with the three dyes

\begin{tabular}{|c|c|c|c|c|c|c|c|c|c|c|c|c|c|}
\hline & $B e$ & $A l$ & $\mathrm{Ca}$ & $T i$ & $\mathrm{Cr}$ & $M n$ & $\mathrm{Fe}$ & Co & $N i$ & $C u$ & $Z n$ & $C d$ & $P b$ \\
\hline $\begin{array}{l}\text { Method 1 } \\
\text { Acid solochrome } \\
\text { azurine (pH 5) }\end{array}$ & Blue & $\begin{array}{l}\text { Blue } \\
\text { purple }\end{array}$ & - & $\begin{array}{l}\text { Deep } \\
\text { purple }\end{array}$ & $\begin{array}{l}\text { Pale } \\
\text { blue }\end{array}$ & - & $\begin{array}{l}\text { Green } \\
\text { blue }\end{array}$ & - & - & Mauve & - & - & - \\
\hline $\begin{array}{l}\text { Method } 2 \\
\text { Aluminon }\end{array}$ & Red & Red & - & - & - & - & $\begin{array}{l}\text { Deep } \\
\text { purple }\end{array}$ & - & - & - & - & - & - \\
\hline $\begin{array}{l}\text { Method } 3 \\
\text { Alkaline solochrome } \\
\text { azurine (pH 8) }\end{array}$ & Purple & $\begin{array}{l}\text { Dark } \\
\text { blue }\end{array}$ & $\begin{array}{l}\text { Pale } \\
\text { orange }\end{array}$ & $\begin{array}{l}\text { Pale } \\
\text { blue }\end{array}$ & Mauve & $\begin{array}{l}\text { Red } \\
\text { brown }\end{array}$ & $\begin{array}{l}\text { Green } \\
\text { blue }\end{array}$ & $\begin{array}{l}\text { Dark } \\
\text { brown }\end{array}$ & $\begin{array}{l}\text { Dark } \\
\text { brown }\end{array}$ & $\begin{array}{l}\text { Royal } \\
\text { blue }\end{array}$ & $\begin{array}{l}\text { Dark } \\
\text { brown }\end{array}$ & Orange & Beige \\
\hline
\end{tabular}

When reproducibility was examined serial sections were always similar with an almost identical distribution and density of final reaction product. Unlike reports of the aluminon method at no time was there an unexplained failure of staining. ${ }^{\circ}$

\section{Discussion}

The staining reaction of method 1 indicated a more extensive distribution of aluminium in compact and trabecular bone than either of the other two methods examined. The presence of a staining reaction by itself, however, does not constitute conclusive evidence for the presence of aluminium. Firstly, the technique is not specific for this metal and, secondly, binding to some non-metallic tissue component could be taking place, although there is no staining in decalcified sections. With atomic absorption spectrophotometry, however, it was shown that all the specimens which stained with method 1 had aluminium in bone trabeculae but not detectable amounts of beryllium, copper, titanium, or chromium, which also react with the dye. With the electron probe technique aluminium but not iron, copper, or titanium could be shown in the bone trabeculae, and the Perl's stain for iron was always negative. These findings do not imply that Perl's positive iron never occurs in bone. On the contrary, we have seen it in conditions of gross iron overload such as haemochromatosis, but it is otherwise rarely seen in bone biopsy specimens and we have yet to see it in patients with dialytic bone disease.

When the electron probe was used to examine sections prestained by method 1 aluminium was detected in the same distribution as the reaction product. None of the patients with normal renal function had concentrations of aluminium in their bone greater than $22 \mu \mathrm{g} / \mathrm{g}$ dried bone and none showed staining by method 1 .

It seems most likely, therefore, that the dye is complexing aluminium, and the dye-aluminium product is well localised to the previous sites of aluminium binding within the bone. While the technique was unable to detect the aluminium known to

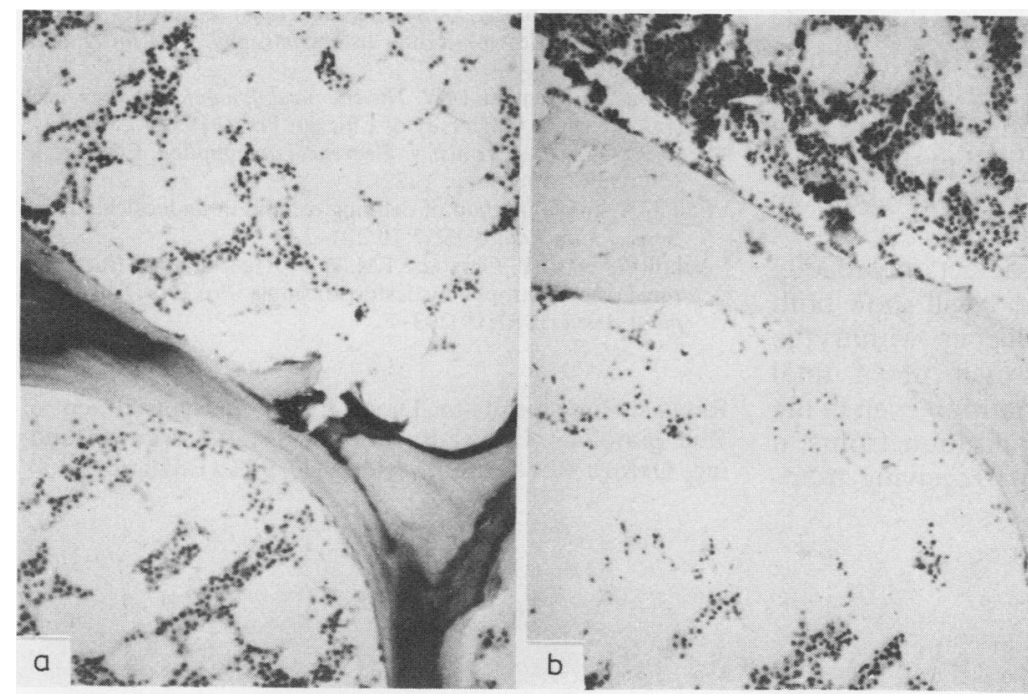

Fig. 5 (a) Intense staining in bone (method 1). (b) Serial section negative for iron in bone (Perl's method). Original magnification $\times$ 100. 
be present in the bone of the non-dialysis patients, it could detect concentrations of aluminium only marginally higher in patients receiving haemodialysis. The probable explanation for this is as follows: although patients with normal renal function might incorporate trace quantities of aluminium, it is distributed throughout the bone; but, perhaps as a result of the combination of high loads, low clearance, and low bone turnover, patients receiving haemodialysis concentrate aluminium in a relatively low volume of bone and local concentrations of aluminium then come within the sensitivity range of method 1. By contrast, spectrophotometric techniques, which measure aluminium as a fraction of total bone mass, are unable to differentiate locally high concentrations in otherwise normal bone from more diffuse patterns of aluminium incorporation. This difference may be important as local high concentrations may be pathologically important whereas diffusely distributed aluminium may not. Despite the focal distribution of aluminium in some patients method 1 offered a rough assessment of total bone aluminium as judged by the results of spectrophotometry.

Method 1 differs from method 3 in using an acid medium; thus the appreciably greater sensitivity of method 1 may depend on the release of aluminium from binding sites within the bone matrix and hence its availability for reaction with the dye-a mechanism perhaps akin to that used in the Perl's reaction for iron. Calcium also reacts with the dye, but the red product, unlike the blue one for aluminium, is alcohol soluble and is thus removed during differentiation.

Method 2, like method 1, employs an acid pH, but we have been unable to stain aluminium at sites other than the calcification fronts using this technique even when the section has been left for $18 \mathrm{~h}$ at room temperature or $40^{\circ} \mathrm{C}$. The reason for this is unclear. If left in the staining solution for $18 \mathrm{~h}$ at $75^{\circ} \mathrm{C}$ (the temperature recommended in the normal staining protocol) the section has always become distorted and impossible to evaluate.

While methods 2 and 3 reliably detect only readily exchangeable aluminium, method 1 will show both this fraction and that bound deeper within the trabeculae. This permits an assessment of total aluminium bone load and its distribution even in the absence of staining at the mineralisation fronts, a pattern we have noted in patients receiving treat- ment for aluminium related bone disease. Although the exchangeable aluminium is possibly the most important pathologically, knowing the total load of aluminium and its distribution may have practical implications in clinical management.

The present method is simple and reliable, but the main advantage is its great senşitivity, allowing the detection of aluminium not only at the fronts but also deep within trabeculae and compact bone. It thus provides a better assessment of the total bone burden and its distribution than other histochemical methods or atomic absorption spectrophotometry. We are at present using method 1 to study the distribution of aluminium and the effects of various forms of treatment on it in a variety of conditions.

We wish to thank Dr P Day, Department of Chemistry, Manchester University, for allowing the use of spectrophotometric data; Mr TC Hopkins and Mr D Plant, Department of Geology, Manchester University, for help in the derivation of electron probe results; and Dr P Ackrill, Withington Hospital, for supplying the biopsy material.

\section{References}

' Pierides AM, Edwards WG, Cullum UX, McCall JT, Ellis HA. Haemodialysis encephalopathy with osteomalacia fractures and muscle weakness. Kidney Int 1980;18:115-24.

${ }^{2}$ Ellis HA, Pierides AM, Feest TG, Ward MK, Kerr DNS. Histopathology of renal osteodystrophy with particular reference to the effects of $1 \alpha$-hydroxyvitamin $D_{3}$ in patients treated by long-term haemodialysis. Clin Endocrinol 1977; 7(suppl):31s-8s.

${ }^{3}$ Buchanan MRC, Ihle BU, Dunn CM. Haemodialysis related osteomalacia: a staining method to demonstrate aluminium. $J$ Clin Pathol 1981;34:1352-4.

${ }^{4}$ Smith PS, McClure J. Localisation of aluminium by histochemical and electron probe X-ray microanalytical techniques in bone tissue of cases of renal osteodystrophy. J Clin Pathol 1982;35: 1283-93.

5 Neuman WF, Neuman MW. The chemical dynamics of bone mineral. Chicago: University of Chicago Press, 1958:

- Pearse AGE. Histochemistry: theoretical and applied. Edinburgh: Churchill Livingstone, 1972:

${ }^{7}$ Ball J. A simple method of defining osteoid in undecalcified sections. J Clin Pathol 1957;10:281-2.

${ }^{8}$ Ackrill P, Day JP, Garstang FM, et al. Treatment of fracturing renal osteodystrophy by desferrioxamine. Pro Eur Dial Transplant Assoc 1982;19:203-7.

Requests for reprints to: Dr AJ Freemont, Department of Rheumatology, University of Manchester, Stopford Building, Oxford Road, Manchester M13 9PT, England. 